\title{
THE EFFECT OF BLEOMYCIN SCLEROTHERAPY IN MANAGEMENT OF INTRA-OSSEOUS HEMANGIOMAS OF THE MANDIBLE
}

\author{
Abdelbadia Abdelmabood ${ }^{*}$ and Ahmed Naguib ${ }^{* *}$
}

\begin{abstract}
Aim: The purpose of this study was to determine the effect of Bleomycin (BLM) sclerotherapy intralesional injection in intraosseous hemangioma of the mandible (IHMs).

Patients and methods: This study was conducted on 7 child patients 5 females and 2 males, their ages ranged from 9 to 12 years of with a mean of 10.4 years, the patients were collected from both the Oral \& Maxillofacial Surgery department Faculty of Dentistry, Tanta. Suez Canal University and Zagazig University Hospital from the period of JUN 2017 to AUGUST 2021. Bleomycin (BLM) agent was evaluated according to its clinical effect on the lesions. And its radiographic effect at the follow-up period by CT scan.

Results: Five cases showed the occurrence of complete response, and two Cases showed the occurrence of marked improvement after, intralesional injection of BLM. Clinically no pain had been detected at the end of the follow-up period, no ulceration had been detected at the site of injection, no bleeding had been detected from the lesion whether spontaneously nor with slight touch in all cases. Orthopantograms and CT Scan and showed an increase in radiopacity of the lesion and a decrease of the lesion cavity size in all cases both after 3 and 6 months from BLM injection.
\end{abstract}

Conclusions: Bleomycin is a safe and cost-effective drug, as a sclerosing agent in the management of child intraosseous hemangiomas.

KEYWORDS: Bleomycin (BLM), Intraosseous hemangiomas of the mandible (IHMs), Computerized Tomogram (CT).

\footnotetext{
* Assistant Professor, Oral Maxillofacial Surgery Department, Faculty of Dental and Oral Surgery; Zagazig University ** Assistant Professor, Oral \& Maxillofacial Surgery, Faculty of Dental and Oral Surgery, Zagazig University.
} 


\section{INTRODUCTION}

Hemangioma is a Greek word composed of Hema means blood, Angio describes vessel and Oma means tumor. Hemangiomas are benign vascular tumors of the blood vessel.(1) The lower jaw is commonly affected three times as common as the upper jaw with a high tendency of affection of the region of the body of the mandible more than the regions of the ramus, condyle and posterior region of the mandible ${ }^{(2)}$.

Epidemiologically, hemangiomas are the most common pediatric tumor; intraosseous hemangiomas (IHMs) commonly occur in the first three decades of life, with female predilection female / male ratio $=5: 1^{(3)}$.

The diagnosis of (IHMs) is challenging, as it shows a wide range of presentations. The definite diagnosis of (IHMs) can be achieved by clinical, histological, and radiographical examination. (4) Clinically, (IHMs) have a characteristic clinical life cycle consisting of three stages including rapid proliferation phase (6-8 months), plateau phase (10-12 months), and slow spontaneous involution phase (5-10 years). Histologically, (IHMs) in the proliferation phase is characterized by the presence of rapidly proliferating endothelial cells lining, the inner aspect of blood vessels, with high turn-over fate. In the involution phase, there is thinking of the vascular endothelial cell lining with the occurrence of peril-vascular deposition of fibro-fatty tissues ${ }^{(5)}$.

IHMs are commonly diagnosed by their typical history and clinical and histological appearance. However, ultrasonography (USG) may be needed in some cases of children aged 2 to 4 years to investigate the main arterial feeding vessel. A computerized Tomogram (CT) is the exact radiograph to study the extensions of (IHMs) ${ }^{(6)}$.

Management of (IHMs) depends on close observation till spontaneous involution occurs. However, this may result in the occurrence of incomplete disappearance of hemangiomas ${ }^{(7)}$. Although most hemangiomas resolve spontaneously, active management is indicated in certain cases such as lesions of the oral cavity, glottic and subglottic locations, particularly in lesions associated with coagulation disorders ${ }^{(\mathbf{8})}$.

Systemic and intralesional corticosteroids (Prednisone) with $3 \mathrm{mg} / \mathrm{kg} /$ day have been used. However, it is not considered as the superior treatment option due to the occurrence of adrenal suppression, Cushing features, and occurrence of both soft tissue muscle, skin, and bone degeneration at the sites of intralesional injection with subsequent esthetic deformities in many cases ${ }^{(\mathbf{9})}$.

The role of the use of Beta-blockers (Propranolol) as a modality for performing the treatment of hemangiomas has been first discovered by Léautélabrèze (2008). However, the exact mechanism of action of oral propranolol in treating pediatric hemangiomas was unclear ${ }^{(\mathbf{1 0})}$.

Surgical excision of the (IHMs) may be considered as the classic treatment, particularly in the small and localized lesions. However, intraoperative and even post-operative hemorrhage may complicate this modality of treatment ${ }^{(11)}$. Therefore the complications of surgery have been the driving force to search for a new treatment option for these vascular lessons.

Sclerotherapy is considered as one of the optimal solutions for performing treatment of (IHMs). This can be achieved by injection of a sclerosing agent into the lumen of the blood vessel or the surrounding interstitial space, leading to the occurrence of endothelial cell injury, fibrous changes thrombosis, scaring, and ultimately occlusion of the blood vessels $^{(\mathbf{1 2})}$

To date, the safety and effectiveness in performing reduction to the growth of (IHMs) remain the main factor in the selection of the suitable sclerosing agent. Recently, intralesional bleomycin injection 
has been proved its effectiveness as an optimal treatment modality for hemangiomas ${ }^{(13,14)}$.

\section{Aim of the study:}

The objective of this study was to determine the efficiency of intralesional Bleomycin injection (BLM) in the management of intraosseous hemangioma of the mandible( IHMs).

\section{PATIENTS OF METHODS}

This prospective randomized study was conducted on seven child patients, suffering from the presence (IHMs), 2 males and 5 females, their ages ranged from 9 - 12 Years from January 2018 to August 2021, with a mean of 10.4 years. The patients that were included in this study were collected from both the Oral \& Maxillofacial surgery department, Faculty of dentistry-Tanta university and Oral \& Maxillofacial surgery department Suez Canal University, and Zagazig University Hospital.

All the details of the treatment procedure, its benefits, potential risks, and the whole study protocol were explained to the parents of the participants in this study and all this were signed in each patient's written consent that describes the details of the procedure.

The inclusive criteria were the presence of (IHMs) of a child up to 12 years who have not received any type of sclerotherapy before.

\section{A-Preoperative assessment}

Demographic data of each patient included the name sex, age, and anatomical location inside the mandible was collected through the study period.

\section{I- History taking}

By asking the patients and his/her parents about, facial asymmetry if was observed, bleeding either spontaneously or with minor trauma, duration of the presence of the lesion, character of pain, associated complications with the presence of (IHMs).

\section{II-Clinical examination:}

Proper intraoral and extraoral clinical examination was performed by both inspection and palpation to detect the presence of bleeding oozing in the suspected area, bony lingual or buccal expansion related to the lesion, nerve injury and numbness, presence of mobility of the related teeth, color change in the mucosa or of the area of the lesion.Fig (1)

\section{III-Radiographic examinations}

1) Preoperative angiographic examination was necessary for preoperative to detect the feeding vessel of the lesion. Fig (2)

2) Computerized Tomograms (CT) scan with threedimensional reconstruction was performed to determine the size and shape of the lesion and to detect the presence of resorption of the roots of the related teeth and also detect whether the (IHMs) affect the related area fig ( 3 -5)

\section{B-Operative procedures:}

- All patients were operated under local anesthesia by inferior alveolar nerve blocks by Mepivacaine $\mathrm{Hcl} 3 \% *$ was injected, to anesthetize the area of the lesion.

- The site of injection was scrubbed with concentrated Betadine** and draped with surgical disposable towels. The body weight of each patient was measured in each treatment session, to determine the amount of sclerosing agent to be injected.

- Bleomycin (BLM) is available from its manufacturer in Bleocel*** 15Iu vials (fig);

\footnotetext{
*Alexandria Co. For Pharmaceutical and Chemical industries Egypt.

** providence iodine $10 \%$, the Nile Co, for Pharmaceuticals and chemicals industries, Cairo, A.R.E.

***Bleocel, CELON , Laboratories, Ltd, India.
} 


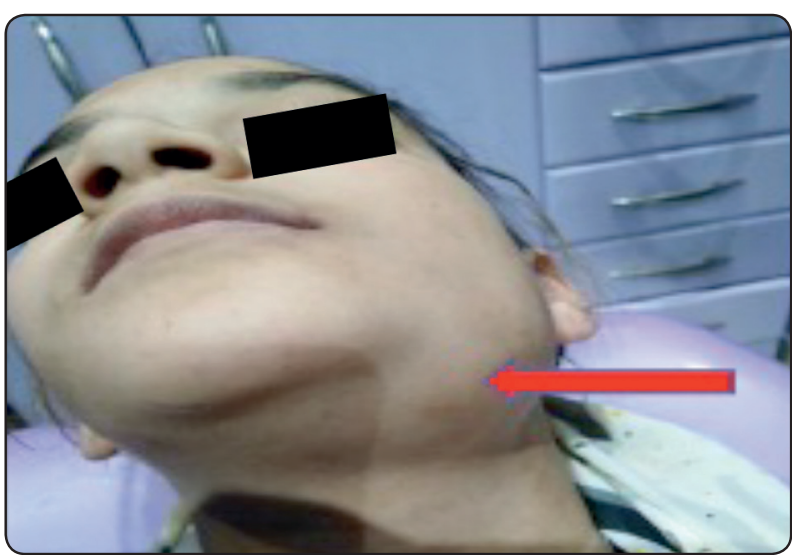

Fig. (1) Preoperative clinical picture (IHMs)

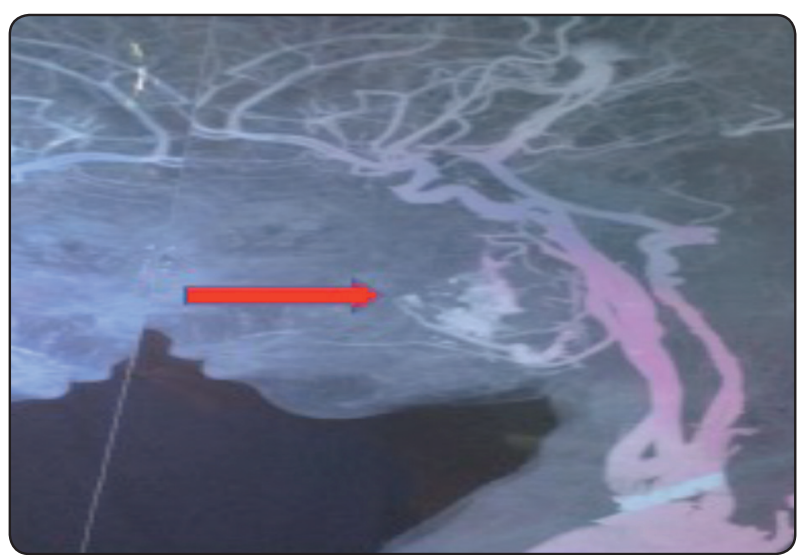

Fig. (2) Preoperative angiograph of (IHMs)

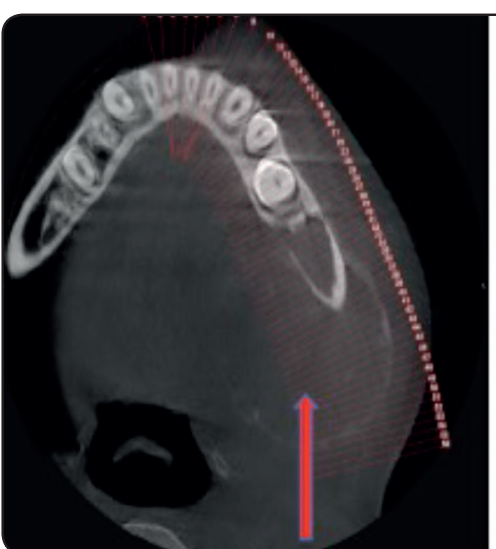

Fig. (3) (IHMs) Axial preoperative CT

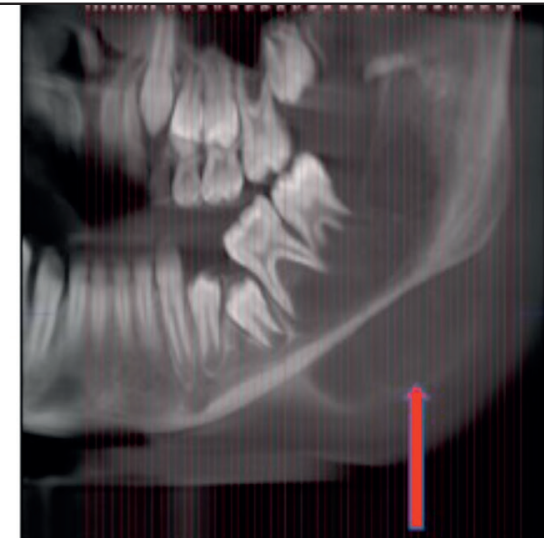

Fig. (4) Coronal Preoperative CT of (IHMs)

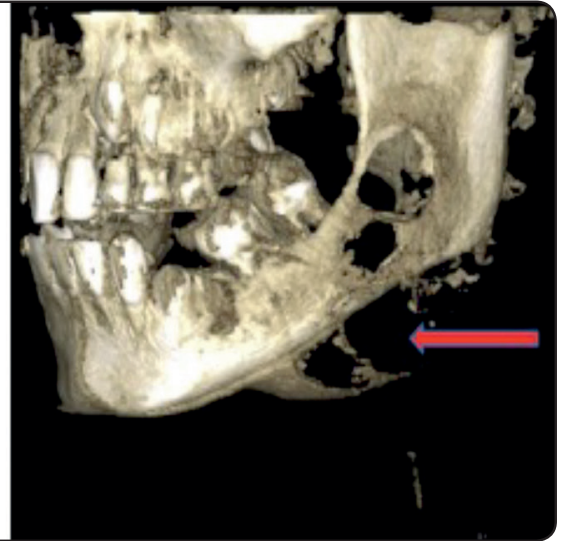

Fig. (5) 3D reconstruction of (IHMs)
BLM vials are stored away from light and at a temperature $2^{\circ}-8^{\circ} \mathrm{c}$. The $15 \mathrm{mg}$ powdered BLM (inside the 15Iu Vial) was dissolved in $15 \mathrm{ml}$ of normal saline $0.9 \%$, BLM is mixed at the dilution of $19 \mathrm{mg} / \mathrm{ml}$ by normal 0.9 salines. The required dose was calculated according to the patient weight. Fig (6)

- The prepared concentrated of injectable BLM solution was slowly injected into the interstitial spaces of the lesion and submucosal after different aspirations before injection of BLM. Fig (7)

- Multiple punctures may be done in the same session intralesional if the lesion contains several non-communicating cavities depending on the radiographic extension of the lesion.

- Repeated intralesional BLM injection can be done up to 8 sessions, at 4 Weeks intervals, and the number of sessions was determined according to each lesion response ${ }^{(15)}$.

- All patients required 6 sessions of BLM sclerotherapy with 4 Weeks intervals, except case number (5) that needed a session of bleomycin sclerotherapy also with 4 Weeks intervals.

\section{Postoperative Management}

- All patients were given $19 \mathrm{mg}$ Ceftriaxone sodium* 19m/Twice-daily intravenously, for 3

* Kohira Pharmaceuticals and chemicals industries Co. Egypt. 


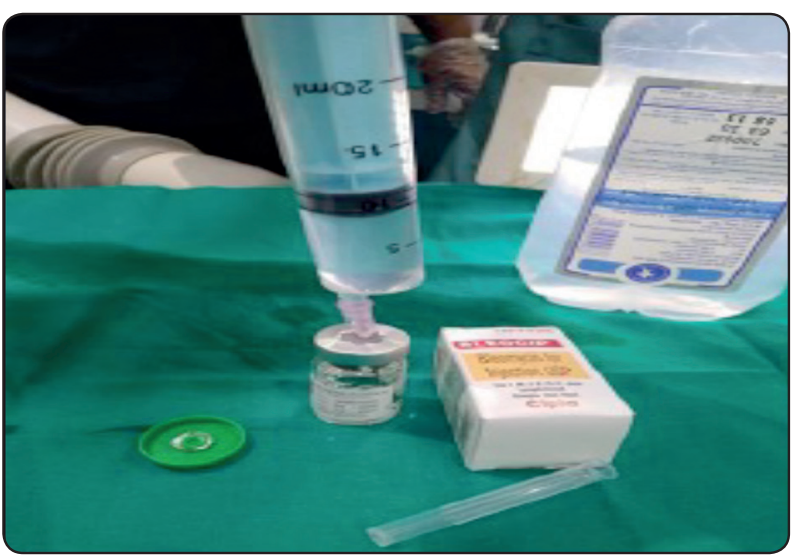

Fig. (6) Preparation of BLM for injection intratesional injection.

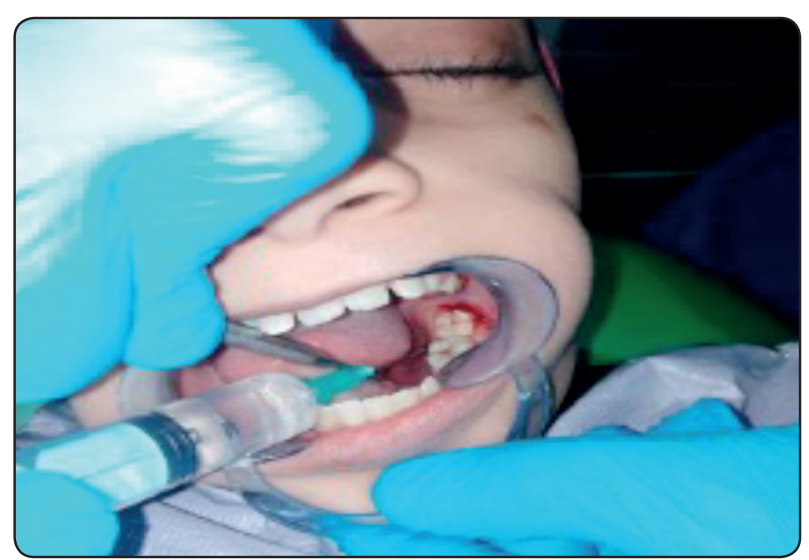

Fig. (7) Intralesional injection of BLM

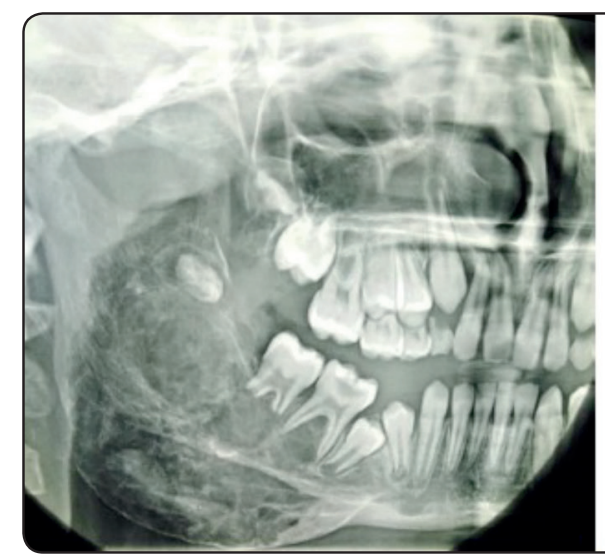

Fig. (8) Postoperative 6 monthe panoramic view

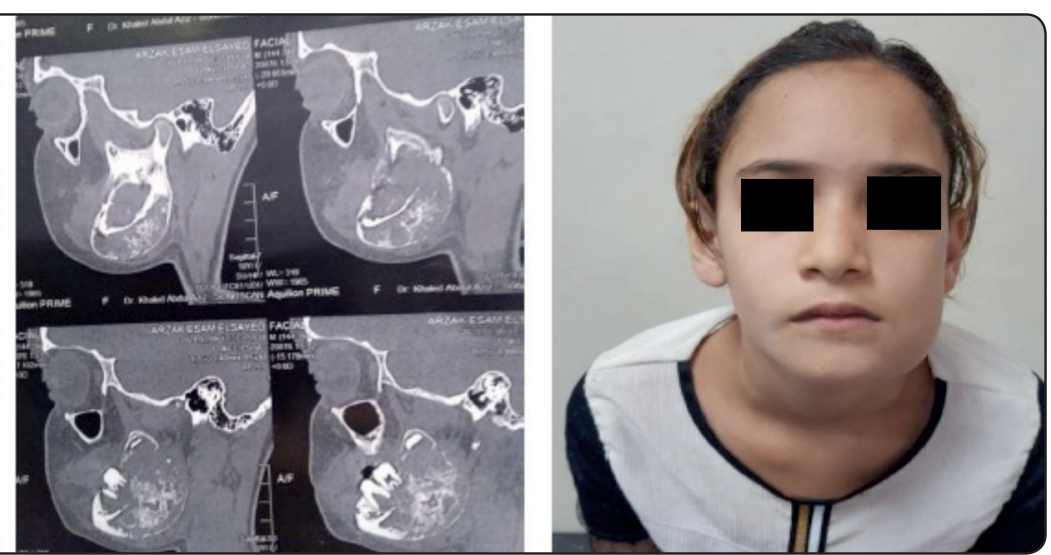

Fig. (9) Postoperative 9 monthe CT

Fig. (10) Postoperative clinical picture days postoperatively. All patients were given $50 \mathrm{mg}$ diclofenac potassium** intramuscularly twice daily for 3 days postoperatively. Cold application prescribed in the first 24 hours, to reduce swelling followed by warm application on the next day.

- All patients were instructed to maintain proper oral hygiene by performing proper teeth brushing and performing oral irrigation with a mixture of saline $0.9 \%$ and povidone-iodine, 4 times/day.

\section{Postoperative assessments}

Postoperative clinical assessment was performed immediately after BLM injection session based on detection of changes in the color of the skin or mucosa, related to the area of the lesion after each session of BLM injection, presence of bleeding with from the lesson whether spontaneously after BLM injection, presence of ulceration at the site of injection, determine the degree of postoperative pain according to VAS scale, presence of postoperative swelling and detect any elevation in the body temperature.

\section{II- Postoperative Radiographic Assessment:}

Postoperative radiographic assessment, at the time intervals of follow-up 3 months CT scan view, 6 months panoramic view fig (8), and 9 months CT scan fig (9) to assess the overall size of the lesions after BLM sclerotherapy

** Egyptian international Pharmaceutical industries Co. 10th of Ramada, City, Egypt 
TABLE (1) Showed demographics and clinical results of BLM Sclerotherapy in all treated patients of the study

\begin{tabular}{ccclcc}
\hline $\begin{array}{c}\text { Base } \\
\text { no }\end{array}$ & $\begin{array}{c}\text { Age } \\
(\text { years })\end{array}$ & sex & \multicolumn{1}{c}{ Site } & $\begin{array}{c}\text { No of } \\
\text { session }\end{array}$ & Treatment outcome \\
\hline 1 & 9 & Female & The left body region of the mandible & 6 & Complete improvement \\
\hline 2 & 11 & Male & The left angle of the mandible & 6 & Complete improvement \\
\hline 3 & 10 & Female & RT body region of the mandible & 6 & Marked improvement \\
\hline 4 & 9.5 & Female & RT body region of the mandible & 6 & Complete improvement \\
\hline 5 & 10.5 & Male & The left angle of the mandible & 7 & Complete improvement \\
\hline 6 & 12 & Female & The left body, angle region of the mandible & 6 & Marked improvement \\
\hline 7 & 11 & Female & The right body region of the mandible & 6 & Complete improvement \\
\hline
\end{tabular}

\section{RESULTS}

Depending on the clinical outcomes and the postoperative radiographic assessment, treatment response was categorized as complete response (>90\% reduction), marked improvement $(>70 \%$ reduction), moderate improvement (40-70\%) reduction, slight improvement $(<40 \%$ reduction $)$ and - no response ${ }^{(15)}$.

From the results of our study there were clinical response 5 cases $(71.4 \%)$ out of 7 Patients had complete response $>90 \%$ improvement fig (10), and only 2 cases (28.6\%) had marked improvement response $>70 \%$ improvement according to the following parameters :

- In the examination of the degree of the post of bleeding from the lesion, no bleeding had been detected from the lesion whether spontaneously not with a slight during BLM sclerotherapy.

- Changes in the color of the skin of the mucosa, related to the area of the lesion, the area of the lesion become comparable with the surrounding areas in all cases.

- Lesion consistency, all cases becomes became firm inconsistency. Presence of ulceration, at the site of injection of BLM, only in the case of cases $2 \& 7$ after the $2^{\text {nd }}, 3^{\text {rd }}$, and $4^{\text {th }}$ session of sclerotherapy. However, it was subsided after cessation of BLM sclerotherapy after 3 months.

- Postoperative pain according to VAS, was scored at the time of BLM injection one day, 3 days, and 7 days after injection. The mean of VAS of BLM injection was $87.03 \pm 4.55$ on the day of BLM injection during the same session of all patients. VAS assessment on 3 days after BLM session injection was decreased where the mean of VAS was 79.80 \pm 5.30 ( P-value 0.001). VAS assessment on 7 days after BLM session injection was decreased where the mean of VAS was $62.60 \pm 8.30$ ( P-value 0.001). over all the time of one month after BLM injection, the pain was decreased with just slight pain owned in case (7) after one month of BLM sclerotherapy.

- Postoperative swelling, no post BLM therapy swelling had been detected in all cases. The presence of elevation in the body temperature, no elevation to in the body temperature had been detected in all cases, after sessions of sclerotherapy.

- Postoperative radiographic assessment, At the time intervals of follow-up 3 months CT scan view, 6 months panoramic view, and 9 months CT scan to assess the overall size of the lesions after BLM sclerotherapy, there was increased the 
radiopacity at the follow-up periods according to the different scans of CT.

\section{DISCUSSION}

Although most hemangiomas disappear spontaneously, however, some indications require active management of these lesions, particularly in large lesions, this agreed with Las Jaunias ${ }^{(8)}$ 1997, who reported that active treatment for hemangiomas may be needed particularly in large lesions at oral cavity which is associated with cardiac overload or coagulation disorder.

Management of hemangiomas can be achieved effectively by sclerotherapy, via intralesional injection of Bleomycin, this was in acceptance by Carqueja et. $\boldsymbol{a l}^{(16)} 2018$ who reported that sclerotherapy had gained popularity as the first line of treatment of hemangiomas.

In our study, regarding the need for anesthesia, all patients were injected with nerve block anesthesia, this was accepted by $\boldsymbol{c h i n}$ et. $\boldsymbol{a l}^{\left(\mathbf{( 1 7 )}^{7}\right.}$ 2003, who reported that only $26.7 \%$ of cases needed nerve block anesthesia.

In our findings, no bleeding had been detected from the lesson whether, spontaneously or with an injection of BLM in session intervals in all cases at the end of the follow-up period, this was in acceptance with Colletti et. $\boldsymbol{a l}^{(\mathbf{1 8})}, \mathbf{2 0 1 5}$, who reported the role of antiangiogenetic drugs in the treatment of vascular lesions, by inhibiting the vascular endothelial growth

Our results demonstrated no postoperative swelling had been detected in all treated cases at the end of the follow-up. This agreed with Mack et al . ${ }^{(19)} 2018$, Who reported all patients in the BLM group did not feel pain after intralesional BLM injection nor suffered from postoperative swelling, due to the reduced acute Inflammatory response of tissues at the site of infection.

During BLM Intra lesion injection, there was no postoperative fever in all treated cases, these results

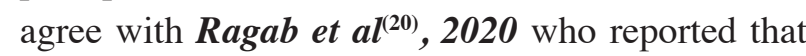

No elevation in the body temperature has been recorded in all treated cases, through the follow-up periods.

Our findings explained, the mean of VAS of BLM injection was $87.03 \pm 4.55$ on the day of BLM injection during the same session of all patients. VAS assessment on 3 days after BLM session injection was decreased where the mean of VAS was 79.80 \pm 5.30 ( P-value 0.001). VAS assessment on 7 days after BLM session injection was decreased where the mean of VAS was $62.60 \pm 8.30$ ( P-value 0.001). Overall of our study after BLM injection, the pain was minimized and no signs of numbness. These findings were in agreement with Eliot and Castle ${ }^{(21)} 2010$, in their study of six cases of the intraosseous vascular lesion with no pain and numbness of five cases of the study.

Our study demonstrated that the 7 cases were located at the mandibular body angle area in the agreement of Gomez et $\mathbf{a l}^{(22)} \mathbf{2 0 0 8}$, as there findings that the most common site of intraosseous vascular anomalies was located in the mandibular posterior area

In our findings, the diagnosis and follow up of all cases depended on panoramic view and computed tomography (CT scan) that explained the accepted results of decreasing the size of the cavitation and increasing the radiopacities over all the CT scans at the follow-up intervals at the time intervals of follow-up 3 months CT scan view, 6 months panoramic view, and 9 months CT scan. This was in agreement with Xiuling et $\boldsymbol{a l}^{(23)}$ 2017, who determined that the diagnosis and follow-up of intraosseous malformation are mainly based on a CT scan.

In addition in our study, we used in the followup of 6 months, an orthopantomogram to determine the expansion of lesions and formation of primary islands of ossification. This was in agreement with. Colletti et $\boldsymbol{a l}^{24} \mathbf{2 0 1 4}$, demonstrated that the initial diagnosis of intraosseous vascular anomalies sometimes is complicated due to their nonspecific image. In their study the cases of intraosseous 
vascular lesions resembling cystic lesions and they recommended overall the follow-up, CT scans should be the ideal that helps to correlate the size of the cavitation about the cortex and the surrounding soft tissue.

\section{REFERENCES}

1. Yan AC, Treat JR, Liange MG. (2010): Terminology used to describe vascular anomalies. Arch pediatr Adolesc Med. 164(2):203-205.

2. Neville BW, Damn DD, chi Ac and Allen CM. (2015): Oral and Maxillofacial Pathology, $4^{\text {th }}$ Edition Philadelphia; Saunders / Elsevier.; 4:123-125

3. Kaban LB, Mulliken JB_(1986) : Vascular anomalies of the maxillofacial region, J Oral Maxillofac Surg., 44 (3); 203-213.

4. Srinivasa R.c., Eleanor Chen, Timothee cousin Dolphina oda.(2017): A case series of intraosseous hemangiomas of the Jaws: various presentations of a rare entity. J. Clin Exp Dent; 9(11): 1336-1370.

5. Theologie Lygidakis N, Schoinohoriti O, Tzermpos F, (2015): Case report review of 6 cases, J Oral Maxillofac Res., 6 (2) : DOI: 105037/jomr.6205.

6. Mulliken MD and Glowacki JG(1982). Hemangiomas and vascular malformations in infants and children: A classification based on endothelial characteristics.Plast Reconstr Surg. 69 (3):412-422.

7. Enjolras O, Mulliken JB. (1997): Vascular tumors and vascular malformations. Adv Dermatol; 13:375-423.

8. Las Jaunias P. (1997): vascular diseases in infants, neonates, and children. $1^{\text {st }}$ New York: Springer-Verlag Berlin Heidelberg.

9. Emir S, Gurlek Gokcebay D, Demirel F, Tunc B. (2015): Efficacy and safety of intralesional corticosteroid application for hemangiomas. Turk J Med Sci. ; 45 (2): 335-338.

10. Leaute-Labreze C , Dumas de la Roque E and Thmbo JB. (2008): Propranolol for sever hemangiomas of infancy. N Engl J Med.;358 (24): 2649-2651.

11. Perkins JA, Manning SC, Tempero RM, et al.(2010): Lymphatic malformations: a review of current treatment. Otolaryngol Head Neck Surg. 142(6)795-803.

12. Casanova D, Boon LM, Vikkula M.(2006): Venous malformation, clinical characteristics, and differential diagnosis. Ann Chir Plast.Esther, 51(5)37-387.
13. Lee HJ, Kim TW and Kim (JM, (2017): percutaneous Sclerotherapy, using bleomycin For the treatment of vascular malformations. Int J Dermatol. ; 56 (11) : 11861191.

14. Kapadia SR, Thakore VM, Patel HM.(2017): Vascular malformation an update on classification, clinical features, and management principles. Indian J Vasc Endovasc Surg. $4: 152-162$.

15. Sainsbury DC, Kessel G, Muir T, Hampton FJ (2011): Intralesional bleomycin injection treatment for vascular birth anomalies a 5-year experience at a single United Kingdom Unit. Plast Reconstr Surg.127:2031- 2044

16. Carqueja IM, Sousa J, Mansilha A(2018): Vascular malformations classification, diagnosis, and treatment. Int Angiol 37(2):127-142

17. Chin KL, Yagiela JA, Quinn CL, Henderson KR(2003): Serum mepivacaine concentrations after intraoral injection in young children. J Calif Dent Assoc 31(10): 757-764

18. Colletti G. Dolmante P, Moneghini L, Ferrari D., and Allevi F, (2015): Adjuvant role of anti-angiogenetic drugs in the management of head and neck arteriovenous malformations. Med Hypotheses; 85: 298-302.

19. Mack JM, Richter GT, Becton D, Salem O, Hill SE(2018): Short term side effects and patient-reported outcomes of bleomycin sclerotherapy in vascular malformation. Pediatr Blood Cancer 65(6).

20. Ragab M, Arafat S, Mohamed W, Aboulhasan M, Eltagy G(2020): intralesional Ethanolamine Oleate injection for management of venous malformation, case series. Indian J Public Health 11(3): 1686-1691.

21. Eliot CA, Castle JT(2010). Intraosseous hemangioma of the anterior mandible. Head Neck Pathol. 2010;4:123-25.

22. Gómez Oliveira G, García-Rozado A, Luaces Rey $\mathrm{R}(2008)$. Intraosseous mandibular hemangioma. A case report and review of the literature. Med Oral Patol Oral Cir Bucal.;13:E496-8.

23. Xiuling H, Jiang A, Yi Z, Zhigang (2017): Intraosseous venous malformation of the zygoma. Reports of 4 cases and literature review. Ann. of Oncology and Laryngology vol 126 (6) 505-509.

24. Colletti G, Frigerio A, Giovanditto F, Biglioli F, Chiapasco M, Grimmer JF(2014). Surgical treatment of vascular malformations of the facial bones. J Oral Maxillofac Surg. 2014 Jul; 72(7): 1326.e1-18. [Medline: 24768420] [doi: 10.1016/j.joms.2014.02.012] 